\title{
A espiritualidade/religiosidade como desafio ao cuidado integral: aspectos regulatórios na formação médica brasileira
}

\author{
I ${ }^{1}$ Gabrielle Cordeiro Trofa, ${ }^{2}$ Ana Claudia Camargo Gonçalves Germani, \\ ${ }^{3}$ Janaine Aline Camargo de Oliveira, ${ }^{4}$ Jose Eluf Neto I
}

Resumo: Apesar das evidências apontarem que a abordagem da espiritualidade/religiosidade na prática clínica está relacionada à redução da mortalidade, à melhora da qualidade de vida e da saúde mental, a falta de treinamento é referida pelos médicos como uma barreira para que tal abordagem aconteça. No Brasil, existem marcos legais que ordenam a formação em saúde, ainda pouco estudados. O objetivo deste estudo foi mapear como documentos normativos da formação médica (de graduação e pós-graduação) abordam a dimensão espiritual/religiosa. Trata-se de estudo qualitativo descritivo exploratório do tipo análise documental, sendo considerados documentos: as Diretrizes Curriculares Nacionais (DCN) do curso de Medicina, os Projetos Políticos Pedagógicos (PPPs) de 20 universidades federais selecionadas e as resoluçóes do Conselho Nacional de Residência Médica em vigor sobre os requisitos mínimos dos Programas de Residência Médica (PRM). A dimensão espiritual/religiosa não foi mencionada nas DCN de medicina, esteve presente em metade dos PPPs analisados e apenas em um programa de residência médica, a Psiquiatria.

> Palavras-chave: Espiritualidade em saúde; Educação Médica; Integralidade em Saúde; Competência Cultural; Controle Social Formal.

\author{
${ }^{1}$ Universidade de São Paulo. São \\ Paulo-SP, Brasil (gabrielle.trofa@ \\ fm.usp.br). \\ ORCID: 0000-0001-5017-5560 \\ ${ }^{2}$ Universidade de São Paulo. São \\ Paulo-SP, Brasil (accggermani@ \\ usp.br). \\ ORCID: 0000-0002-7409-915X \\ ${ }^{3}$ Universidade de São Paulo. São \\ Paulo-SP, Brasil (jancamargo@ \\ hotmail.com). \\ ORCID: 0000-0002-2415-2979 \\ ${ }^{4}$ Universidade de São Paulo. São \\ Paulo-SP, Brasil (jelufnet@usp.br). \\ ORCID: 0000-0001-7504-2115
}

Recebido em: 04/12/2019 Aprovado em: 06/04/2020 Revisado em: 14/10/2021 


\section{Introdução}

Estudos vem demonstrando que a espiritualidade e a religiosidade de um indivíduo podem influenciar no seu processo saúde-doença-cuidado, na medida em que estão relacionadas à redução da mortalidade (HUMMER et al., 1999; CHIDA et al., 2009), bem como à melhora da qualidade de vida (SAWATZKY et al., 2005; UNTERRAINER et al., 2014; SHERMAN et al., 2015) e da saúde mental (LUCCHETTI et al., 2011; GONÇALVES et al., 2015; AKRAWI et al., 2015; KOENIG, 2015; LAWRENCE et al., 2016). Além disso, estudos demonstram que grande parte dos pacientes tem interesse em discutir espiritualidade no contexto do atendimento em saúde (EHMAN et al., 1999; MACLEAN et al., 2003; MCCORD et al., 2004).

Desse modo, a literatura aponta para a importância da abordagem da espiritualidade/religiosidade na prática clínica. Nesse sentido, a Associação de Universidades Médicas Americanas (AAMC), a Organização Mundial da Saúde e a Comissão Conjunta sobre Acreditação de Organizações de Saúde (JCAHO) há anos recomendam que questôes espirituais sejam abordadas em atendimento clínico e na educação de profissionais de saúde (PUCHALSKI e LARSON, 1998). $\mathrm{O}$ posicionamento das sociedades médicas também vem crescendo, a exemplo da Academia Americana de Médicos de Família e Comunidade, que defende que já há evidências suficientes para indicar a abordagem da espiritualidade na prática clínica e que deixar de fazê-lo poderia ser considerado má prática (ANANDARAJAH e HIGHT, 2001). Do mesmo modo, a Associação Mundial de Psiquiatria também já se posicionou a respeito da importância da abordagem do tema no cuidado em saúde mental (MOREIRA-ALMEIDA et al., 2016).

Diante desse contexto, o desenvolvimento da competência para a abordagem da dimensão espiritual/religiosa do paciente por parte dos médicos torna-se necessário para o desempenho de uma prática clínica que olhe o processo saúde-doença-cuidado de modo adequado. Por outro lado, pesquisas realizadas com médicos americanos (MCCAULEY et al, 2005; CURLIN et al, 2006) e brasileiros (OLIVEIRA, 2018) apontam que, dentre as principais barreiras enumeradas pelos profissionais médicos como impedimentos para a abordagem da espiritualidade na prática clínica, destacam-se a falta de tempo e a falta de conhecimento ou treinamento na área. Nesse sentido, surge a necessidade de investigar como o ensino e o desenvolvimento 
dessa competência permeiam a formação médica durante a graduação e a residência médica nas diferentes especialidades atualmente, no Brasil.

De acordo com os dados da Demografia Médica no Brasil, em novembro de 2017, havia 289 escolas médicas em atividade (que somavam 29.271 vagas), além de 16 cursos autorizados pelo Governo Federal que naquele momento ainda não haviam definido o início de funcionamento e do número de vagas a serem ofertadas. De acordo com o site (ESCOLAS MÉDICAS DO BRASIL, 2020), o número atualizado em janeiro de 2020 era de 341 escolas médicas em funcionamento no Brasil, correspondendo a 35.258 vagas.

As Diretrizes Curriculares Nacionais (DCN) do Curso de Graduaçáo em Medicina são um documento norteador para a organização, desenvolvimento e avaliação do Curso de Medicina, no contexto dos sistemas de ensino superior do país. Estabelecem os princípios, os fundamentos e as finalidades da formação em Medicina, bem como estabelecem as competências a serem desenvolvidas pelo egresso do curso. A mais recente DCN do curso de medicina foi instituída em 2014 pela Câmara da Educação Superior (CES) do Conselho Nacional de Educação (CNE).

Em parecer CNE/CES no 116/2014 consta que a implementação das diretrizes curriculares deverá contribuir para a inovaçáo e para a qualidade do projeto pedagógico, devendo orientar o currículo do Curso de Graduação em Medicina para um perfil profissional do egresso condizente com as políticas de saúde do país. Assim, as DCN devem nortear a elaboração do Projeto Político Pedagógico do Curso de cada faculdade, apresentando grande relevância na formação médica em nível de graduação.

O panorama relacionado aos profissionais já formados, com dados de janeiro de 2018, menciona 451.777 registros de médicos em atividade no País, sendo que 62,5\% (282.298) têm um ou mais títulos de especialista, enquanto 37,5\% (169.479) não têm título algum (SCHEFFER, 2018).

A residência médica é uma modalidade de ensino de pós-graduação destinada a médicos, sob a forma de curso de especialização, instituída pelo Decreto no 80.281, de 5 de setembro de 1977. Funciona em instituições de saúde, sob a orientação de profissionais médicos de elevada qualificação ética e profissional, sendo considerada o "padrão ouro" de especialização médica.

No Brasil, os programas de residência médica são regulamentados pela Comissão Nacional de Residência Médica (CNRM), instância colegiada de caráter consultivo 
e deliberativo do Ministério da Educação e tem a finalidade de regular, supervisionar e avaliar as instituiçóes e programas de residência médica. (BRASIL, 2011, decreto $\mathrm{n}^{\circ}$ 7.562, de 15 setembro de 2011)

A CNRM é responsável por estabelecer os requisitos mínimos dos programas de residência médica, os quais constituem documentos regulamentadores dos programas de residência médica do Brasil. Assim, esses documentos são de grande importância para nortear a formação dos profissionais das diversas especialidades médicas, descrevendo as competências exigidas dos profissionais em formação e, consequentemente, orientando a prática clínica.

De acordo com a legislação, a CRNM é composta por 12 conselheiros titulares e 12 suplentes, que representam diferentes instituições (Ministério da Educação, Ministério da Saúde, Conselho Nacional de Secretários de Saúde - CONASS; Conselho Nacional de Secretários Municipais de Saúde - CONASEMS; Conselho Federal de Medicina - CFM; Associação Brasileira de Educação Médica - ABEM; Associação Médica Brasileira - AMB; Associação Nacional de Médicos Residentes - ANMR, Federação Nacional de Médicos - FENAM; Federação Brasileira de Academias de Medicina - FBAM). Importante mencionar ainda a Câmara Técnica e as Comissões Estaduais de Residência Médica - CEREM, unidades descentralizadas da CNRM nos Estados e no Distrito Federal como instâncias auxiliares.

A qualidade dos programas de residência médica e as competências por eles almejadas na formaçáo do profissional influenciam a qualidade da prática clínica dos diversos especialistas formados e em atuação. Isso está diretamente envolvido no atendimento recebido pelo usuário, sua satisfação e seu processo saúde-doença-cuidado.

A ordenação da formação de recursos humanos na área da saúde está prevista nos marcos legais do SUS (CONSTITUIÇÃO FEDERAL, Artigo 200 e Lei 8.080, Artigo $6^{\circ}$ ). Uma das formas de ordenação se dá pela regulação legal e infralegal. A regulação das profissôes da saúde é uma atividade estatal que envolve a formação (ensino técnico, graduação e especialização), o exercício profissional (registro, ética, competências legais, atividades permitidas) e também as relaçóes de trabalho (jornada de trabalho, salários, carreiras, etc.) (AITH, 2019).

Cientes da polissemia que envolve o assunto de regulação e, sobretudo das consequências para a análise de políticas públicas, entende-se que a regulação, na perspectiva do direito, seja composta pelo estabelecimento e implementação de regras e normas e manutenção ou restabelecimento do equilíbrio de um sistema. Defende- 
se ainda que os marcos regulatórios incluam os mecanismos de controle social, uma vez que expressam a participaçáo da comunidade organizada em conselhos no processo decisório sobre políticas públicas de formação (OLIVEIRA, 2014).

Apesar de sua extensão prática, a produção teórica na literatura nacional sobre aspectos regulatórios ainda é incipiente, sobretudo no que se refere a formação médica. Frente a necessidade do desenvolvimento da competência cultural para abordagem da dimensão espiritual/religiosa do paciente pelo médico, este estudo visa identificar se a competência da abordagem espiritual/religiosa está presente em três instâncias. Primeiramente, nas Diretrizes Curriculares Nacionais (DCN) do curso de Medicina. Em segundo lugar, nos Projetos Político Pedagógicos de cursos de medicina de universidades federais e por fim, entre requisitos mínimos dos Programas de Residência Médica da Comissão Nacional de Residência Médica (CNRM) especificando em quais especialidades. Espera-se com isso contribuir e fortalecer documentos norteadores da formação médica, em direção à integralidade das dimensóes do cuidado.

\section{Objetivo}

Mapear se os documentos normativos da formação médica (de graduação e pósgraduação) abordam a dimensão espiritual/religiosa e em caso positivo, descrever como é feita tal abordagem.

\section{Métodos}

Foi realizado estudo qualitativo descritivo exploratório do tipo análise documental, sendo adotado na análise o paradigma construtivista, no qual as interpretaçóes são reconhecidamente subjetivas e socialmente construídas. Foram considerados como documentos: a DCN do curso de graduação em Medicina publicada em 2014, os PPPs de cursos de medicina e as resoluçóes do CNRM em vigor sobre os requisitos mínimos dos Programas de Residência Médica (PRM) das especialidades médicas reconhecidas no Brasil e os requisitos mínimos de seus programas em vigor em dezembro de 2018.

Considerando a dimensão nacional das DCNs e dos PRM, optou-se por selecionar os PPPs das universidades federais que constituem 23\% (78) das escolas médicas no Brasil e 2\% (7006) das vagas de $1^{\circ}$ ano (ESCOLAS MÉDICAS DO 
BRASIL, 2020). A partir deste recorte, foram selecionadas quatro escolas de cada uma das cinco regiôes geográficas brasileiras. Por entender a importância da dimensão histórica na construção do PPP, foram elencadas dentro de cada região, as duas instituiçôes mais antigas e as duas mais recentes que disponibilizassem seus PPPs nos respectivos websites.

Em relação à residência médica no Brasil, a portaria do CME no $1 / 2018$, homologada pelo CFM na resolução no 2.221/2018, é o documento mais recente com a relação de especialidades e áreas de atuação médica e reconhece 55 especialidades no Brasil, que serão apresentadas nos resultados.

Os requisitos mínimos dos programas de residência médica dessas especialidades são determinados pela CNRM e podem ser obtidos através do site do Ministério da Educação (MEC), na página de Residência Médica, onde há Ementário da Residência Médica - (Leis - Decretos e resolução - Em vigor e revogadas). Nesta página há as resoluçóes da CNRM e a respectiva ementa. Para a etapa pré-analítica, ou seja, a fase de organização, o acesso aos documentos se deu nos meses de agosto e setembro de 2017, sendo realizada a seleção das ementas em vigor relacionadas aos requisitos mínimos dos programas de residência médica, com a atenção para aquelas especialidades que tiveram atualizações em seus requisitos mínimos em ementas posteriores.

Para a exploração dos dados, foi feita leitura exaustiva das DCNs do curso de medicina, dos PPPs dos cursos de medicina selecionados bem como das ementas e requisitos mínimos de cada especialidade em busca de três categorias definidas a priori:

1) Menção direta ou indireta à competência da abordagem espiritual.

2) Menção direta ou indireta à abordagem da religiosidade, embora conceitualmente diferente da espiritualidade;

3) Menção a uma abordagem mais ampliada ou atenção integral da saúde.

\section{Resultados}

O texto das DCN publicado em 2014 e produzido pela Câmara de Educação Superior do Conselho Nacional de Educação tem 14 páginas e trata da atenção integral, sem citar diretamente a espiritualidade/religiosidade:

Art. $5^{\circ} \mathrm{Na}$ Atenção à Saúde, o graduando será formado para considerar sempre as dimensões da diversidade biológica, subjetiva, étnico-racial, de gênero, orientação sexual, socioeconômica, política, ambiental, cultural, ética e demais aspectos que compóem o espectro da diversidade humana que singularizam cada pessoa ou cada grupo social. (Brasil, $2014 \mathrm{pl}$ ) 
Em relação aos PPPs das 20 escolas de medicina selecionadas, observa-se que em 10 (50\%) há menção direta à abordagem da espiritualidade/religiosidade do paciente, enquanto as demais fazem uma abordagem indireta do tema, na medida em que trazem uma abordagem integral à saúde (Tabela 1).

Tabela 1. Projetos Políticos Pedagógicos de 20 faculdades federais de medicina e abordagem da espiritualidade/religiosidade (E/R), 2020

\begin{tabular}{|c|c|c|c|c|}
\hline Regiáo & Sigla & $\begin{array}{l}\text { Ano da } \\
\text { criaçáo }\end{array}$ & $\begin{array}{l}\text { Ano do } \\
\text { PPP }\end{array}$ & Abordagem da E/R \\
\hline \multirow{4}{*}{ Centro-Oeste } & UFG & 1960 & 2017 & Sim (espiritualidade) \\
\hline & UFMS & 1967 & 2014 & Abordagem integral \\
\hline & UFMS - Três Lagoas & 2014 & 2019 & $\begin{array}{c}\text { Sim (espiritualidade) - disciplina } \\
\text { optativa }\end{array}$ \\
\hline & UFT - Araguaína & 2015 & 2019 & Abordagem integral \\
\hline \multirow{4}{*}{ Nordeste } & UFBA & 1808 & 2010 & Abordagem integral \\
\hline & UFPE & 1920 & 2019 & Abordagem integral \\
\hline & UFPI - Parnaíba & 2014 & 2014 & Sim (espiritualidade) \\
\hline & UFAL - Arapiraca & 2015 & 2018 & $\begin{array}{c}\text { Sim (espiritualidade }) \text { - disciplina } \\
\text { optativa }\end{array}$ \\
\hline \multirow{4}{*}{ Norte } & UFPA & 1919 & 2010 & Abordagem integral \\
\hline & UFAM & 1965 & 2009 & Abordagem integral \\
\hline & UNIFAP & 2010 & 2016 & Sim (religiosidade) \\
\hline & UFAM - Coari & 2017 & 2014 & Abordagem integral \\
\hline \multirow{4}{*}{ Sudeste } & UNIRIO & 1912 & 2014 & Sim (religiosidade) \\
\hline & UFJF & 1952 & 2019 & Sim (religiosidade) \\
\hline & UNIFAL & 2013 & 2015 & Sim (espiritualidade) \\
\hline & $\begin{array}{l}\text { UFVJM - Teófilo } \\
\text { Otoni }\end{array}$ & 2014 & 2018 & Sim (espiritualidade) \\
\hline
\end{tabular}




\begin{tabular}{|c|c|c|c|c|}
\hline Regiáo & Sigla & $\begin{array}{l}\text { Ano da } \\
\text { criaçáo }\end{array}$ & $\begin{array}{l}\text { Ano do } \\
\text { PPP }\end{array}$ & Abordagem da E/R \\
\hline \multirow{4}{*}{ Sul } & UFSM & 1954 & 2016 & Abordagem biopsicossocial \\
\hline & UFPR & 1912 & 2019 & Abordagem integral \\
\hline & $\begin{array}{l}\text { UNIPAMPA - } \\
\text { Uruguaiana }\end{array}$ & 2015 & 2019 & $\begin{array}{c}\text { Sim (espiritualidade) }- \text { disciplina } \\
\text { optativa }\end{array}$ \\
\hline & UFPR - Toledo & 2018 & 2018 & Abordagem integral \\
\hline
\end{tabular}

Por ordem na tabela: UFG: Universidade Federal de Goiás, UFMS: Universidade Federal de Mato Grosso do Sul, UFMS - Três Lagoas: Universidade Federal de Mato Grosso do Sul Campus Três Lagoas, UFT - Araguaína: Universidade Federal de Tocantins Campus de Araguaína, UFBA: Universidade Federal da Bahia, UFPE: Universidade Federal de Pernambuco, UFPI - Parnaíba: Universidade Federal do Piauí Campus de Parnaíba, UFAL - Arapiraca: Universidade Federal de Alagoas Campus de Arapiraca, UFPA: Universidade Federal do Pará, UFAM: Universidade Federal do Amazonas, UNIFAP: Universidade Federal do Amapá, UFAM - Coari: Universidade Federal do Amazonas Campus Médio Solimōes Coari, UNIRIO: Universidade Federal do Estado do Rio de Janeiro, UFJF: Universidade Federal de Juiz de Fora, UNIFAL: Universidade Federal de Alfenas, UFVJM - Teófilo Otoni: Universidade Federal dos Vales do Jequitinhonha e Mucuri Campus Teófilo Otoni, UFSM: Universidade Federal de Santa Maria, UFPR: Universidade Federal do Paraná, UNIPAMPA - Araguaiana: Universidade Federal do Pampa Campus Araguaiana, UFPR - Toledo: Universidade Federal do Paraná Campus Toledo.

Entre os 20 PPPs analisados, três foram elaborados antes de 2014, ano da versão vigente das DCN. A abordagem integral presente no documento norteador dos cursos esteve presente em todos os PPPs, sendo que sete explicitam a espiritualidade e três tratam da religiosidade. Destaca-se ainda que todos os quatro cursos da região Sudeste abordam explicitamente a dimensão da espiritualidade/religiosidade e apenas um da Região Norte. Entre os cursos mais antigos (criados entre 1808 e 1967), apenas um incorporou a temática em seu PPP.

No PPP da UFG, há a menção à "promoção do conforto físico, emocional e espiritual”, habilidade que deve ser aprendida pelo estudante em alguns dos módulos do curso. No PPP da UNIRIO, também consta o tema no currículo nuclear, neste caso na disciplina de "Bioética e ética médica", fazendo referência à religiosidade. $\mathrm{Na}$ UFJF, a religiosidade está presente no contexto de abordagem da saúde integral da população negra. Já no PPP da UFVJM - Teófilo Otoni, a palavra-conceito aparece no item de metodologia de ensino. A UFP - Parnaíba traz o tema nos objetivos do curso: "valoriza o paciente em todos os seus aspectos (biológico, psíquico, social e espiritual)”. Na UNIFAL, a espiritualidade em cuidados paliativos é citada no curso 
sobre habilidades de comunicação e humanidades em medicina. Já nos PPPs da UFAL - Arapiraca, UFMS - Três Lagoas e UNIPAMPA - Uruguaiana, consta a existência de disciplinas optativas sobre saúde e espiritualidade.

Nas resoluções do CNRM, no contexto das residências médicas, apenas uma especialidade - psiquiatria - menciona diretamente a espiritualidade/religiosidade e cinco trazem uma abordagem biopsicossocial ou atenção integral: endoscopia, ginecologia e obstetrícia, medicina de família e comunidade, medicina do trabalho e pediatria.

A Resolução da CNRM no 2 de 17 de maio de 2006 é a mais antiga resolução em vigor que dispóe sobre requisitos mínimos dos PRM. Apenas as especialidades de Oncologia Clínica, Cirurgia da Mão, Medicina de Família e Comunidade (MFC) e Pediatria tiveram seus requisitos mínimos alterados em resoluçóes posteriores.

A leitura exaustiva desse documento permitiu identificar que não há um padrão de texto dos requisitos mínimos para as especialidades. Assim, para algumas especialidades o texto dá enfoque na porcentagem da carga horária que deve ser destinada em cada serviço (ambulatório, enfermaria, etc), enquanto textos de outras especialidades focam nos temas a serem abordados em cada ano da residência e outros ainda abordam as competências a serem alcançadas pelos profissionais que passam pelos programas de residência.

Nessa resolução, apenas no trecho referente à especialidade de Psiquiatria, há menção à espiritualidade/religiosidade. Segundo o texto, uma das competências a serem alcançadas, presente no item 9, é:

"O residente demonstrará conhecimento do crescimento e do desenvolvimento humano, incluindo os desenvolvimentos biológico, cognitivo e psicossexual normais, bem como os fatores socioculturais, econômicos, étnicos, sexuais, religiosos/espirituais e familiares.”

Em nenhuma outra especialidade há mençáo direta ou indireta à abordagem espiritual/religiosa. Realizando uma leitura atenta, podemos identificar que em algumas especialidades há a menção a uma abordagem mais ampliada de saúde, como no trecho do texto referente à Endoscopia: “ [...] com capacidade de avaliar o paciente de forma abrangente em seu todo biopsicossocial [...]”. Esse texto ainda se mantém em vigor, já que na resolução da CNRM no 8 , de 30 de outubro de 2006, houve a alteração apenas dos pré-requisitos para realização do programa de residência em Endoscopia. 
No texto referente aos requisitos mínimos dos PRM de Medicina de Família e Comunidade, há também menção à “abordagem biopsicossocial”, expressa no trecho: "[...] formar um especialista cuja característica básica é atuar, prioritariamente, em Atenção Primária à Saúde, a partir de uma abordagem biopsicossocial do processo saúde adoecimento [...]”. Esses requisitos não estão mais em vigor, na medida em que foram atualizados em resolução posterior que será aqui mencionada.

A resolução da CNRM no 1 , de 25 de maio de 2015, regulamenta os requisitos mínimos dos PRM em Medicina de Família e Comunidade, atualizando o texto até aquele momento em vigor. $\mathrm{O}$ texto possui uma abordagem ampla e cita as competências a serem desenvolvidas ao longo da residência, além dos fundamentos da especialidade, objetivos do programa, entre outros tópicos. Não menciona, entretanto, a espiritualidade/religiosidade. Também não faz referência a uma abordagem biopsicossocial, apesar de entre os fundamentos da especialidade estar "comprometer-se com o cuidado integral às pessoas sob sua responsabilidade [...]".

A abordagem do cuidado integral também está presente nos textos referentes à Medicina do Trabalho e a Obstetrícia e Ginecologia. Na primeira especialidade está presente no trecho: "[...] atenção integral à saúde dos trabalhadores, compreendendo ações de promoção e proteção da saúde, prevenção de doença, diagnóstico, tratamento e reabilitação”. Já na segunda especialidade, está contida no trecho: “[...] atenção integral à gravida adolescente: acompanhamento da gestante adolescente durante o pré-natal, enfocando a fisiologia do desenvolvimento normal da gestação e do concepto às necessidades médicas, psicológicas e sociais da adolescente [...]”, no qual também há referência a uma abordagem biopsicossocial.

Os requisitos mínimos do PRM em Pediatria também sofreram alteraçóes, as quais se deram pela resolução da CNRM no 1 , de 29 de dezembro de 2016. Neste documento, são citados além da distribuição da carga horária, os conhecimentos e competências esperados para cada ano da residência. No entanto, entre as diversas competências ali presentes, não há menção à competência da abordagem espiritual/ religiosa. Apesar disso, a abordagem biopsicossocial é citada no trecho referente a um dos conhecimentos e competências a serem desenvolvidas no primeiro ano da residência: "reconhecer a importância das condiçôes ambientais, psicológicas e socioculturais no atendimento de crianças e adolescentes". Além disso, também há a menção do cuidado integral entre as habilidades e atitudes a serem desenvolvidas no segundo ano de residência: "prestar atendimento integral à saúde do adolescente". 
Assim, pode-se notar que, apesar de para algumas especialidades haver a menção de uma abordagem biopsicossocial ou atenção/cuidado integral, apenas no texto referente à Psiquiatria há menção à espiritualidade/religiosidade.

\section{Discussão}

Apesar do texto das DCN não abordar explicitamente a dimensão da espiritualidade/religiosidade, metade dos PPPs analisados avançam nesta direção. Foram observadas diferenças regionais e também em relação ao tempo de existência da universidade. Tal resultado demonstra a diversidade nos programas oferecidos, como prevê o texto geral da DCN (BRASIL, 2001, parecer CNE/CES no 583, de 4 de abril de 2001). Ao mesmo tempo, defende-se que a variação e flexibilidade dos programas deve buscar melhor atender às necessidades e peculiaridades das regióes, neste sentido a dimensão espiritual/religiosa deveria estar presente.

A escassez da menção à competência da abordagem da dimensão espiritual/ religiosa nos documentos regulamentadores da formação médica observada no presente estudo contribui para o debate sobre a falta de estudos brasileiros que lidam com a incorporação da espiritualidade/religiosidade na educação médica.

Uma revisão bibliográfica (LUCCHETTI et al., 2012b) indica que há predominância de estudos relacionados a saúde/medicina e espiritualidade nos cursos médicos nos EUA e no Canadá. Os estudos encontrados na revisão abordam temas como a inserção da espiritualidade em cursos de educação médica, a incorporação em cursos de residência médica e especialização, maneiras de incorporar a espiritualidade nos currículos médicos, número de escolas médicas que possuem cursos que abordam a espiritualidade na graduação, e aspectos espirituais de estudantes de medicina, residentes e professores. Foi apontado que $75 \%$ das escolas médicas dos EUA fornecem ensino sobre questôes religiosas e espirituais com aplicação à medicina (PUCHALSKI, 2006). Outras pesquisas apontam que 90\% das escolas de medicina dos EUA (KOENIG et al., 2010) e 59\% das escolas de medicina britânica (NEELY e MINFORD, 2008), possuem cursos ou conteúdo em espiritualidade e saúde.

Já no contexto brasileiro, em um estudo de Lucchetti et al. (2012a) sobre o ensino de espiritualidade e saúde nas escolas médicas no Brasil, observou-se que menos da metade fornece alguma forma de ensino sobre o assunto e poucas escolas (10,4\% das 
86 escolas pesquisadas) têm cursos que abordam especificamente a espiritualidade e a saúde, sendo que, em quatro escolas privadas, o conteúdo foi desenvolvido em disciplinas obrigatórias e nas 5 demais, públicas, em disciplinas eletivas. Entretanto, a maioria dos diretores médicos (54\%) acreditam que esta questáo é um assunto importante que deve ser ensinado.

Estudo mais recente (CONDE et al., 2019) investigou a presença formal da espiritualidade nos currículos das escolas médicas da regiáo Norte do Brasil, bem como a perspectiva dos internos de medicina sobre a importância da espiritualidade como parte do cuidado integral ao ser humano. Observou-se que das dezesseis escolas médicas selecionadas, apenas duas, ambas privadas, apresentavam a temática da espiritualidade no currículo das suas instituiçôes. Em relação à perspectiva do estudante de medicina, $70,6 \%$ concordam que a espiritualidade deveria fazer parte dos currículos dos cursos de medicina.

Em um estudo de Lucchetti et al. (2013), foi encontrado similar resultado. Dos estudantes de doze escolas médicas que participaram do estudo, 71,2\% acreditam que a espiritualidade tem impacto na saúde do paciente. A maioria também gostaria de abordar espiritualidade/religiosidade na prática clínica (58\%). No entanto, em relação ao preparo para abordagem do tema, quase metade $(48,7 \%)$ se sentem despreparados. Em relação ao treinamento para tal abordagem, $81 \%$ referem nunca terem participado de uma atividade sobre saúde e espiritualidade.

Esses estudos evidenciam que a temática da espiritualidade/religiosidade não está presente na maioria dos currículos das escolas médicas, comprometendo a formação do estudante para abordar tal tema no contexto de cuidado ao paciente. Isso encontra paralelo nos resultados aqui encontrados, em que 50\% das escolas médicas selecionadas por esse estudo não mencionam a abordagem da espiritualidade/ religiosidade em seus PPPs.

Já a literatura sobre a incorporação da temática de espiritualidade/religiosidade e saúde durante a residência médica é ainda escassa e evidencia um cenário que requer muitos avanços. Nos EUA, houve a implementação de cursos de espiritualidade e saúde em programas de residência em medicina interna, psiquiatria e medicina de família. (PUCHALSKI, 2006). Entretanto, muitos avanços ainda são necessários. Em um estudo realizado nos EUA com a avaliação do treinamento em espiritualidade e cuidado em saúde nos programas de residência médica de medicina de família, 
aproximadamente $92 \%$ dos diretores dos programas disseram ser o ensino da espiritualidade importante. No entanto, apenas $31 \%$ dos programas têm um currículo específico (com média de 6 horas) para ensinar espiritualidade e cuidado em saúde para seus residentes (KING e CRISP, 2005).

Comparado ao progresso do tema em outros países, o reconhecimento da importância da espiritualidade e religiosidade para a saúde, bem como de sua abordagem na prática médica não parece estar sendo suficiente para promover mudanças nos documentos regulamentadores dos PRM no Brasil. Isso pode impactar na garantia da efetiva abordagem do tema nos programas de residência médica.

Para além das análises aqui mencionadas, o presente estudo permitiu observar que as resoluções da CNRM que dispóem sobre os requisitos mínimos dos programas de residência médica das especialidades reconhecidas no Brasil não possuem um padrão do texto, de modo que há divergências do conteúdo entre as especialidades. Assim, nem todas as especialidades explicitam as competências a serem desenvolvidas ao longo da formação profissional. Além disso, a maioria das especialidades não teve seu texto atualizado desde a resolução de 2006 em vigor. Dessa forma, não ocorreu uma adaptação às necessidades evidenciadas na atualidade.

Faz-se necessário pontuar que o artigo tem como base a análise documental de textos produzidos entre 2006 e 2019, logo os resultados tratam da realidade vigente até 2019, ou seja, são datados. Sabe-se, por exemplo, da dinâmica dos PPPs e também que o CNRM aprovou em novembro de 2018 Matrizes de Competências para 45 especialidades. De toda forma, ciente das rupturas e permanências envolvidas no processo regulatório, o resultado do presente artigo mapeia a abordagem da espiritualidade/religiosidade em documentos nacionais e de universidades federais. Documentos de outra natureza, como os PPPs de universidades estaduais e privadas, bem como programas específicos elaborados pelas instituiçóes que oferecem residência médica poderiam acrescentar novos elementos a discussão. Ressalta-se ainda que os achados referentes aos PPPs são trazidos para exemplificar a operacionalização das DCNs pelas instituiçóes e não pretendem ser generalizáveis, uma vez que a amostra selecionada não busca ser representativa do universo dos cursos existentes.

Como já mencionado, a falta de padrão estabelecido para o conteúdo dos textos sobre os requisitos mínimos dos PRM das diversas especialidades, faz com que haja variação de conteúdo e formato entre os textos das especialidades. Outro fator 
limitante é que a abordagem da dimensão espiritual/religiosa seria uma necessidade que pode estar embutida em outras competências mais amplas, como o cuidado biopsicossocial, podendo limitar que o termo "religiosidade" ou "espiritualidade" seja explicitado na descrição das competências.

Como observado em outras análises de políticas públicas (ALESSSIO et al, 2016; COSTA et al 2017), é essencial reforçar e defender a potência dos marcos regulatórios, construídos de forma democrática. Entende-se que tais documentos sejam uma forma de promover e assegurar a melhoria progressiva da formação em saúde, incorporando evidências e corroborando para a oferta do cuidado integral preconizada no SUS.

Cabe lembrar que o SUS prevê e assegura a participação social e o controle social como forma dos cidadãos participarem das políticas públicas. Entende-se ainda que tais políticas são dinâmicas - mudam e se transformam - a partir de embates entre atores implicados (SILVA e Baptista, 2014). Como os documentos analisados são produzidos e aprovados por Conselheiros de naturezas distintas, espera-se que a participação represente, em última instância, os interesses da população. Como apontado por Aith (2019) "Zelar para que o interesse público prevaleça é eixo estruturante para assegurar o direito a saúde e a consolidação do SUS”.

\section{Conclusão}

Considerando as três instâncias de documentos analisados, podemos afirmar que a competência da abordagem da dimensão espiritual/religiosa ainda é pouco abordada nos documentos norteadores gerais, sendo observada em documentos locais. O fato de a competência cultural da abordagem da dimensão espiritual/ religiosa do paciente não ser mencionada nas DCN de medicina e ser citada apenas nos requisitos mínimos de Psiquiatria evidencia que os avanços no conhecimento da importância da abordagem espiritual/religiosa na prática clínica não estão tendo reflexo em documentos que regulamentam a educação médica no Brasil. A presença da dimensão espiritual/religiosa nos PPPs mostra a potência de marcos norteadores locais. De toda forma, sugere-se entáo que os espaços institucionalizados, responsáveis pela formulação, monitoramento e avaliação de tais documentos, estimulem e garantam o debate sobre o tema, buscando a incorporação e a consolidação de melhorias progressivas na formação em saúde, em direção ao cuidado integral. 


\section{Referências}

ALESSIO, M. M.; SOUSA, M.F. Regulação da formação de especialistas: inter-relações com o Programa Mais Médicos. Physis [online]. 2016, 26 (2): 633-667.

AKRAWI, D. A. et al. Religiosity, spirituality in relation to disordered eating and body image concerns: A systematic review. J Eat Disord. 2015;15(3):29.

AITH, F. A. M. O interesse público na regulação estatal de profissóes de saúde do Brasil. Rev. Latino-Am. Enfermagem 2019;27.

ANANDARAJAH, G.; HIGHT, E. Spirituality and medical practice: Using the HOPE questions as a practical tool for spiritual assessment. Am Fam Physician. 2001;63(1):81-8.

BRASIL. Constituição, 1988. Constituição da República Federativa do Brasil 1988.

BRASIL. Diário Oficial da União. Decreto no 7562, de 15 de setembro de 2011. Disponível em: http://portal.mec.gov.br/index.php?option=com_docman\&view=download\&alias=8959decreto-7562-2011\&category_slug=setembro-2011-pdf\&Itemid=30192 Acesso em: 01 set. 2017. BRASIL. Ministério da Educação. Ementário da Residência Médica - Leis - Decretos e resoluçóes - Comissão Nacional de Residência Médica - CRNM. Disponível em: http://portal.mec.gov. br/index.php?option=com_docman\&view=download \&alias=60361-ementario-da-residenciamedica-pdf\&category_slug=fevereiro-2017-pdf\&Itemid=30192 Acesso em: 01 set. 2017.

BRASIL. Ministério da Educação. Parecer CNE/CES no 116, de 3 de abril de 2014. Disponível em: http://portal.mec.gov.br/index.php?option=com_docman\&view=download\&alias=15514pces116-14\&category_slug=abril-2014-pdf\&Itemid=30192. Acesso em: 3 de fev. 2020.

BRASIL. Ministério da Educação. Parecer CNE/CES no 583, de 4 de abril de 2001. Disponível em: http://portal.mec.gov.br/cne/arquivos/pdf/CES0583.pdf. Acesso em: 3 de fev. 2020.

BRASIL. Ministério da Educação. Residências em saúde - residência médica. Disponível: http:// portal.mec.gov.br/residencias-em-saude. Acesso em: 01 set. 2017.

BRASIL. Ministério da Educação. Matrizes de Competências Aprovadas pela CNRM. Disponível em: http://portal.mec.gov.br/component/content/article?id=71531. Acesso em: 17 nov. 2019.

BRASIL. Ministério da Educação. Resolução CNRM no 02, de 17 de maio de 2006. Disponível em: http://portal.mec.gov.br/index.php?option=com_docman\&view=download\&alias=512resolucao-cnrm-02-17052006\&Itemid=30192. Acesso em: 01 set. 2017.

BRASIL. Ministério da Educação. Resolução CNRMno 8, de 30 de outubro de 2006. Disponível em: http://portal.mec.gov.br/index.php?option=com_docman\&view=download\&alias=526resolucao-cnrm-08-30102006\&category_slug=documentos-pdf\&Itemid=30192 Acesso em: 01 set. 2017. 
BRASIL. Ministério da Educação. Resolução CNRM no 1, de 31 de julho de 2007. Disponível em: http://portal.mec.gov.br/sesu/arquivos/pdf/cnrm/resolucao_01_de_2007.pdf Acesso em: 01 set. 2017.

BRASIL. Ministério da Educação. Resolução CNRM no 2, de 20 de agosto de 2007. Disponível em: http://www.cirurgiadamao.org.br/_arquivo/cet/regras_residencia_medica.pdf Acesso em: 01 set. 2017.

BRASIL. Ministério da Educação. Resolução no 3, de 20 de junho de 2014. Institui Diretrizes Curriculares Nacionais do Curso de Graduação em Medicina e dá outras providências. [S. 1.], 20 jun. 2014. Disponível em: http://portal.mec.gov.br/index.php?option=com docman \&view=download \&alias=15874-rces003-14\&category_slug=junho-2014pdf\&Itemid=30192. Acesso em: 01 set. 2017.

BRASIL. Ministério da Educação. Resolução CNRM no 1, de 25 de maio de 2015. Disponível em: http://portal.mec.gov.br/index.php?option=com_docman\&view=download \&alias=17661res01-25052015-sesu-residencia-medica\&category_slug=junho-2015-pdf\&Itemid=30192 Acesso em: 01 set. 2017.

BRASIL. Ministério da Educação. Resolução CNRMño 1, de 29 de dezembro de 2016. Disponível em: http://portal.mec.gov.br/index.php?option=com_docman\&view=download\&alias=55611pediatria-3-anos-pdf\&category_slug=janeiro-2017-pdf\&Itemid=30192 Acesso em: 01 set. 2017. BRASIL. Presidência da República, Casa Civil, Subchefia para Assuntos Jurídicos. Decreto $n$. 7.562, de 15 de setembro de 2011. Dispóe sobre a Comissão Nacional de Residência Médica e o exercício das funções de regulação, supervisão e avaliação de instituiçôes que ofertam residência médica e de programas de residência médica. [S. 1.], 15 set. 2011. Disponível em: http://www. planalto.gov.br/ccivil_03/_Ato2011-2014/2011/Decreto/D7562.htm. Acesso em: 01 set. 2017.

BRASIL. Presidência da República, Casa Civil, Subchefia para Assuntos Jurídicos. Decreto federal $n^{\circ}$ 8.516, de 10 de setembro de 2015. Regulamenta a formação do Cadastro Nacional de Especialistas de que tratam o $\$ 4^{\circ}$ e $\$ 5^{\circ}$ do art. $1^{\circ}$ da Lei no 6.932, de 7 de julho de 1981, e o art. 35 da Lei no 12.871, de 22 de outubro de 2013. [S. 1.], 10 set. 2015. Disponível em: http://www. planalto.gov.br/ccivil_03/_ato2015-2018/2015/decreto/d8516.htm. Acesso em: 01 set. 2017.

BRASIL. Presidência da República, Casa Civil, Subchefia para Assuntos Jurídicos. Decreto $n^{o}$ 80.281, de 5 de setembro de 1977. Regulamenta a Residência Médica, cria a Comissão Nacional de Residência Médica e dá outras providências. [S. 1.], 5 set. 1977. Disponível em: http://portal. mec.gov.br/index.php?option=com_docman\&view=download \&alias=535-decreto-8028105091977\&category_slug=documentos-pdf\&Itemid=30192. Acesso em: 01 set. 2017.

CHIDA, Y.; STEPTOE, A.; POWELL, L. H. Religiosity/spirituality and mortality. A systematic quantitative review. PsychotherPsychosom. 2009;78(2):81-90. 
CONDE, S. R. S. S. et al. A espiritualidade nos currículos das escolas médicas da região norte e a visão do interno de medicina sobre sua importância na formação. Interdisciplinary Journal of Health Education. 2019;4(1-2):9-18

CONSELHO FEDERAL DE MEDICINA. Resolução no 2.221/2018. Homologa a Portaria CME no 1/2018 que atualiza a relação de especialidades e áreas de atuação médicas aprovadas pela Comissão Mista de Especialidades. Disponível em: https://sistemas.cfm.org.br/normas/ visualizar/resolucoes/BR/2017/2162 Acesso em: 17 nov. 2019.

COSTA P.H.A; RONZANI T.M; COLUGNATI F.A.B. "No papel é bonito, mas na prática...” Análise sobre a rede de atenção aos usuários de drogas nas políticas e instrumentos normativos da área. Saúde Sociedade. 2017,26(3):738-750.

CURLIN, F. A. et al. The association of physicians' religious characteristics with their attitudes and self-reported behaviors regarding religion and spirituality in the clinical encounter. Med Care. 2006;44(5):446-53.

ESCOLAS MÉDICAS DO BRASIL. Todas as escolas médicas [S.I.]. Disponível em: https:// www.escolasmedicas.com.br/escolas-medicas-todas.php?orderby=cat_nome\&order=DESC Acesso em: 27 jan. 2020.

KING, D. E.; CRISP, J. Spirituality and Health Care Education in Family Medicine Residency Programs. Fam Med 2005;37(6):399-403.

EHMAN, J. W. et al. Do patients want physicians to inquire about their spiritual or religious beliefs if they become gravely ill? Arch Intern Med 1999;159(15):1803-6.

FORMIGLI, V. L. et al. Projeto Político-Pedagógico do Curso de Graduação em Medicina da FMB/UFBA. Gaz. Méd. Bahia 2010;80:1(Jan-Abr):3-47.

GONÇALVES, J. P. et al. Religious and spiritual interventions in mental health care: a systematic review and meta-analysis of randomized controlled clinical trials. Psychol Med. 2015;45(14):2937-49.

HUMMER, R. A. et al. Religious involvement and U.S adult mortality. Demography 1999;36(2):273-285.

KOENIG, H. G. et al. Spirituality in Medical School Curricula: Findings from a National Survey. Int J Psychiatry Med. 2010,40:391-398.

KOENIG, H. G. Religion, spirituality, and health: a review and update. Adv Mind Body Med. 2015 Summer;29(3):19-26.

LAWRENCE, R. E.; OQUENDO, M. A.; STANLEY, B. Religion and Suicide Risk: A Systematic Review. Arch Suicide Res. 2016;20(1):1-21.

LUCCHETTI, G. et al. Religiousness affects mental health, pain and quality of life in older people in an outpatient rehabilitation setting. J Rehabil Med. 2011;43(4):316-22. 
LUCCHETTI, G. et al. Spirituality and health in the curricula of medical schools in Brazil. BMC Med Educ. 2012a;12(1):78

LUCCHETTI, G.; LUCCHETTI, A. L.; PUCHALSKI, C. M. Spirituality in medical education: global reality? J Relig Health 2012b;51:3-19

LUCCHETTI, G., DE OLIVEIRA, L.R., KOENIG, H.G. et al. Medical students, spirituality and religiosity-results from the multicenter study SBRAME. BMC Med Educ 13, 162 (2013).

MCCAULEY, J. et al. Spiritual beliefs and barriers among managed care practitioners. J Relig Health. 2005 Summer;44(2):137-46.

MACLEAN, C. D. et al. Patient preference for physician discussion and practice of spirituality. J Gen Intern Med. 2003;18(1):38-43.

MCCORD, G. et al. Discussing spirituality with patients: a rational and ethical approach. Ann Fam Med 2004;2(4):356-61.

MOREIRA-ALMEIDA, A. et al. WPA Position Statement on Spirituality and Religion in Psychiatry. World Psychiatry. 2016;15:87-88.

NEELY, D.; MINFORD, E.J. Current status of teaching on spirituality in UK medical schools. Medical education. 2008, 42:176-182.

OliveIRA, J. A. C. Desafios do cuidado integral em saúde: A dimensão espiritual do médico se relaciona com sua prática na abordagem espiritual do paciente? Dissertação de mestrado. Faculdade de Medicina da Universidade de São Paulo. São Paulo. 2018.

OLIVEIRA, R.R. Dos conceitos de regulação às suas possibilidades. Saúde Sociedade. 2014,, $23(4), 1198-1208$.

PUCHALSKI, C. M.; LARSON, D. B. Developing curricula in spirituality and medicine. Academic medicine: journal of the Association of American Medical Colleges. 1998,73:970-974.

PUCHALSKI, C. M. Spirituality and medicine: Curricula in medical education. Journal of Cancer Education, 2006,21(1),14-18.

SAWATZKY, R.; RATNER, P.A.; CHIU, L. A meta-analysis of the relationship between spirituality and quality of life. Soc Indic Res. 2005;72(2):153-88.

SCHEFFER, M. et al, Demografia Médica no Brasil 2018. Departamento de Medicina Preventiva, Faculdade de Medicina da USP. Conselho Regional de Medicina do Estado de São Paulo. Conselho Federal de Medicina. Sáo Paulo: 2018, 286 páginas. ISBN: 978-85-87077-55-4.

SHERMAN, A. C. et al. A meta-analytic review of religious or spiritual involvement and social health among cancer patients. Cancer. 2015;121(21):3779-88.

SILVA, P. F.A; BAPTISTA, T.W.F. Os sentidos e disputas na construção da Política Nacional de Promoção da Saúde. Physis [online]. 2014, 24 (2): 441-465. 
UNIVERSIDADE FEDERAL DE ALAGOAS. Campus de Araripaca. Projeto Pedagógico do Curso de Medicina. 2018. Disponível em: http://www.ufal.edu.br/arapiraca/graduacao/medicina/ projeto-pedagogico/projeto-pedagogico-do-curso-2018/view Acesso em: 27 jan. 2020.

UNIVERSIDADE FEDERAL DE ALFENAS. Projeto Politico Pedagógico do Curso de Medicina. 2015. Disponível em: https://www.unifal-mg.edu.br/graduacao/system/files/imce/ Cursos/Medicina/PPC\%20com\%20Res\%20anexa.\%20Publicado\%2025-01-2016.pdf Acesso em: 27 jan. 2020.

UNIVERSIDADE FEDERAL DE GOIÁS. Faculdade de Medicina. Projeto Pedagógico. 2017. Disponível em: https:/files.cercomp.ufg.br/weby/up/148/o/PPC-2017.pdf?1505222188 Acesso em: 27 jan. 2020.

UNIVERSIDADE FEDERAL DE JUIZ DE FORA. Projeto Politico Pedagógico de Curso Faculdade de Medicina. 2019. Disponível em: http://www.ufjf.br/medicina/files/2015/04/PPCFAMED2019.pdf. Acesso em: 27 jan. 2020.

UNIVERSIDADE FEDERAL DE PERNAMBUCO. Pró-reitoria para assuntos acadêmicos. Centro de Ciências Médicas. Coordenação do curso de Medicina. Projeto Pedagógico do Curso de Graduação em Medicina Campus Recife. 2019. Disponível em: https://www.ufpe.br/ documents/1894452/0/PPC+medicina+2019.2.pdf/f4248618-b13b-4082-a26c-5f04f35d5a76 Acesso em: 27 jan. 2020.

UNIVERSIDADE FEDERAL DE SANTA MARIA. Centro de Ciências da Saúde. Projeto Pedagógico do Curso de Medicina. 2016. Disponível em: https://www.ufsm.br/cursos/graduacao/ santa-maria/medicina/projeto-pedagogico Acesso em: 27 jan. 2020.

UNIVERSIDADE FEDERAL DE TOCANTINS. Conselho de Ensino, Pesquisa e Extensão. Resolução $n^{o}$ 14, de 27 de março de 2019. Dispóe sobre a atualização do Projeto Pedagógico do Curso (PPC) de Medicina (Câmpus de Araguaína) aprovado pela Resolução Consepe no 05/2016. Disponível em: https://docs.uft.edu.br/share/s/pouR7KppSQi6C4PWG82f7g Acesso em: 27 jan. 2020.

UNIVERSIDADE FEDERAL DO AMAPÁ. Pró-reitoria de Ensino de Graduação. Projeto Pedagógico do Curso de Medicina. 2016. Disponível em: https://www2.unifap.br/medicina/ files/2015/05/ppc-atual.pdf Acesso em: 27 jan. 2020.

UNIVERSIDADE FEDERAL DO AMAZONAS. Faculdade de Medicina. Projeto PoliticoPedagógico Medicina. 2009. Disponível em: http://www.home.ufam.edu.br/dcc1/joomla/ Projeto\%20Pedagógico\%20Curso\%20de\%20Medicina.pdf Acesso em: 27 jan. 2020.

UNIVERSIDADE FEDERAL DO AMAZONAS. Campus Médio Solimões - Coari. Coordenação do Curso de Graduação em Medicina. Projeto Pedagógico do Curso de Graduação em Medicina Campus Médio Solimões - Coari. 2014. Disponível em: http://edoc.ufam.edu.br/ bitstream/123456789/2078/8/PPC\%20MEDICINA\%20ISB\%20COARI_16_04_\%202019_ Atualizado.pdf Acesso em: 27 jan. 2020. 
UNIVERSIDADE FEDERAL DO ESTADO DO RIO DE JANEIRO. Centro de Ciências Biológicas e da Saúde. Escola de Medicina e Cirurgia. Projeto Pedagógico do Curso de Medicina. 2017. Disponível em: http://www.unirio.br/emc/arquivos/ppc-medicina-2014.1 Acesso em: 27 jan. 2020.

UNIVERSIDADE FEDERAL DO MATO GROSSO DO SUL. Resolução no 556, de 20 de dezembro de 2014. Disponível em: https://famed.ufms.br/files/2018/12/556.pdf Acesso em: 27 jan. 2020.

UNIVERSIDADE FEDERAL DO MATO GROSSO DO SUL. Resolução no 594, de 8 de novembro de 2019. Aprovar Projeto Pedagógico do Curso de Medicina - Bacharelado do Câmpus de Três Lagoas. Disponível em: https://cptl.ufms.br/files/2019/11/2019__Res_594 Aprova_o_PPC__Completo_.pdf Acesso em: 27 jan. 2020.

UNIVERSIDADE FEDERAL DO PAMPA. Campus Uruguaiana. Projeto Pedagógico do Curso de Medicina Bacharelado. 2019. Disponível em: http://cursos.unipampa.edu.br/cursos/ medicina/files/2019/11/ppc-2019-medicina.pdf Acesso em: 27 jan. 2020.

UNIVERSIDADE FEDERAL DO PARÁ. Instituto de Ciências da Saúde. Faculdade de Medicina. Projeto Pedagógico Curso de Medicina. 2010. Disponível em: http://www. faculdademedicina.ufpa.br/doc/ppc.pdf Acesso em: 27 jan. 2020.

UNIVERSIDADE FEDERAL DO PARANÁ. Setor de Ciências da Saúde. Projeto Pedagógico do Curso de Medicina. 2019. Disponível em: http://www.saude.ufpr.br/portal/medicina/wpcontent/uploads/sites/10/2019/04/PPC_MEDICINA_2019.pdf Acesso em: 27 jan. 2020.

UNIVERSIDADE FEDERAL DO PARANÁ. Campus Toledo. Projeto Pedagógico do Curso de Medicina. 2018. Disponível em: http://www.toledo.ufpr.br/portal/wp-content/ uploads/2017/07/PPC-Medicina-Toledo-2018.pdf Acesso em: 27 jan. 2020.

UNIVERSIDADE FEDERAL DO PIAUÍ. Campus de Parnaíba. Projeto Pedagógico do Curso de Graduação em Medicina. 2014.

UNIVERSIDADE FEDERAL DOS VALES DO JEQUITINHONHA E MUCURI. Campus Mucuri. Projeto Pedagógico do Curso de Graduação em Medicina. 2018. Disponível em: http://site.ufvjm.edu.br/fammuc/files/2019/07/PPC-12-FINAL.pdf Acesso em: 27 jan. 2020.

UNTERRAINER, H. F.; LEWIS, A. J.; FINK, A. Religious/Spiritual well-being, personality and mental health: a review of results and conceptual issues. J ReligHealth. 2014;53(2):382-92. 


\section{Abstract}

\section{Spirituality/religiosity as a challenge to comprehensive care: regulatory aspects in Brazilian medical education}

The aim of this study was to assess the quality of oral care provided in Brazilian primary care and identify associated contextual socioeconomic factors. This is a cross-sectional study whose unit of analysis was all the oral care teams that participated in an external assessment for the second cycle of the Access and Quality Improvement Program - Primary Care. Quality was measured in the following dimensions: access, resolution, work process, care coordination and infrastructure. The quality criteria of the external assessment were grouped into these dimensions and weighted using Item Response Theory. The indicator of oral care quality was the result of grouping latent variables. Descriptive analyses were performed by region and association between "Oral Care Quality" and the Municipal Human Development Index (HDI), with $5 \%$ significance. Only $25 \%$ of the oral care teams were considered good. The infrastructure dimension obtained the best results and care coordination the worst. There was no statistically significant difference between municipalities with high and low HDIs. Quality assessment is vital to ensure the continuous improvement of quality healthcare.

Keywords: Spirituality in medicine; Medical Education; Comprehensiveness care; Cultural Competency; Social Control. 